\title{
REIC - Edição Especial - Artigos do CTIC 2019 Editorial
}

\author{
Marcel Oliveira $^{1}$, Regiane Kawasaki ${ }^{2}$ \\ ${ }^{1}$ Universidade Federal do Rio Grande do Norte \\ Natal - RN - Brasil \\ marcel@dimap.ufrn.br \\ ${ }^{2}$ Universidade Federal do Pará \\ Belém - PA - Brasil \\ marcel@dimap.ufrn.br, kawasaki@ufpa.br
}

Esta edição especial da REIC reúne artigos que apresentam os trabalhos selecionados como os melhores trabalhos realizados por alunos de graduação submetidos ao XXXVIII Concurso de Trabalhos de Iniciação Científica (CTIC 2019) do XIX Congresso da Sociedade Brasileira de Computação (CSBC 2019), que ocorreu em Belém, Pará, de 14 a 18 de Julho de 2018.

O CTIC é um evento anual organizado e promovido pela Sociedade Brasileira de Computação (SBC) e tem como objetivo incentivar alunos de todos os cursos de graduação da área de Computação a produzirem textos científicos originais sobre trabalhos realizados por eles, em temas relacionados à área. O CTIC aceita trabalhos realizados não só por alunos de cursos da área de Computação, como também por alunos de outros cursos que realizaram trabalhos na área.

Nesta edição, o CTIC teve 44 trabalhos registrados, dos quais 39 correspondiam a submissões válidas. Todos os artigos artigos submetidos foram avaliados por três membros do comitê de programa. Com base nesta avaliação, foram selecionados os 10 melhores, que foram convidados a submeter a versão final do artigo para publicação nesta edição. Um desses artigos desistiu da publicação e, por este motivo, esta edição apresenta 9 artigos.

Durante o CSBC 2019, no CTIC 2019, houve uma nova etapa de avaliação. Nesta etapa, os artigos classificados nas 3 primeiras posições (listados abaixo), apresentaram os seus trabalhos perante o comitê de avaliação final.

- ParallelUS: Um Ambiente Paralelo e Distribuição para Aplicações Móveis. Millas Avelar, Guilherme Andrade e Leonardo Rocha.

- Técnicas de redução de dados em redes de sensores sem fio tolerantes a atrasos. Israel L. C. Vasconcelos e Andre L. L. Aquino.

- Simulating Systems-of-Systems Dynamic Architectures. Wallace Manzano, Valdemar Vicente Graciano Neto e Elisa Yumi Nagakawa.

A realização do CTIC 2019 não seria possível sem a dedicação de muitos envolvidos. Primeiramente, todos os autores merecem um agradecimento pela sua submissão e alta qualidade dos trabalhos submetidos. Além disso, merecem agradecimento todos os membros do comitê de programa e do comitê de avaliação final, pelo ótimo trabalho de revisão e avaliação, e a equipe da SBC, pelo suporte na realização do evento. 


\section{Coordenação Geral}

Marcel Oliveira (UFRN)

\section{Coordenação Local}

Regiane Kawasaki (UFPA)

\section{Comitê de Avaliação Final}

- Marcel Oliveira (UFRN)

- Regiane Kawasaki (UFPA)

- Jair Leite (UFRN)

- Renata Galante (UFRGS)

- Carlos Ferraz (UFPE)

\section{Comitê de Programa}

- Alba Melo (UnB)

- Anne Canuto (UFRN)

- Bernadette Loscio (UFPE)

- Bruno Cafeo (UFMS)

- Carla Freitas (UFRGS)

- Charles Madeira (UFRN)

- Claudio Sant'Anna (UFBA)

- Daniel Araújo (UFRN)

- Danielle Couto (UFPA)

- David Menotti (UFPR)

- Eduardo Aranha (UFRN)

- Elder José Cirilo (UFSJ)

- Everton Cavalcante (UFRN)

- Fatima Duarte-Figueiredo (PUC Minas)

- Fernando Trinta (UFC)

- George Teodoro (UFMG)

- Humberto Marques (PUC Minas)

- Igor Wiese (UTFPR)

- Ingrid Nunes (UFRGS)

- Ivanovitch Silva (UFRN)

- Jair Leite (UFRN)

- Jerusa Marchi (UFSC)

- José Viterbo (UFF)

- Juliana Vizzotto (UFSM)

- Leonardo Rocha (UFSJ)

- Luis Rivero (UFMA)

- Marcel Oliveira (UFRN)

- Marcio Ribeiro (UFAL)

- Monica Pereira (UFRN)

- Rafael Melo (UFBA)

- Rafael Torchelsen (UFPel)

- Regiane Kawasaki (UFPA)

- Renata Galante (UFRGS) 
- Ricardo Marroquim (UFRJ)

- Ricardo Santos (UFMS)

- Ricardo Terra (UFLA)

- Rosiane de Freitas Rodrigues (UFAM)

- Sílvia Maia (UFRN)

- Thais Vasconcelos Batista (UFRN)

- Uirá Kulesza (UFRN)

- Vinicius Garcia (UFPE) 